\title{
Characterization of PDE Reducible to ODE under a Certain Homogeneity and Applications to Singular Cauchy Problems
}

\author{
By \\ Takuya WATANABE and Jiichiroh URABE \\ (Ritsumeikan University and Doshisha University, Japan)
}

\begin{abstract}
We give a necessary and sufficient condition for a homogeneous partial differential equation in two variables to be reduced to a homogeneous ordinary one under a certain change of variables. It is described by means of the commutator with a first order partial differential operator which characterizes a homogeneity. Moreover we obtain the explicit representation of the reduced ordinary differential equation. This result is a generalization of such a reduction which had been applied to singular Cauchy problems in our previous works [U, WU1]. This fact suggests that local structures of the solutions to partial differential equations can be described by global structures of those to ordinary ones.

Key Words and Phrases. Commutation relation, Euler's homogeneous function theorem, A singular Cauchy problem, Hypergeometric differential equation.

2010 Mathematics Subject Classification Numbers. 34A25, 35A20, 35G05, $44 \mathrm{~A} 45$.
\end{abstract}

\section{Contents}

1. Introduction and results 226

1.1. Framework of the reduction from PDE to ODE 226

1.1.1. Strategy of the reduction from PDE to ODE 227

1.1.2. Characterization of reducible partial differential operators 229

1.1.3. Succession of singularities from PDE to ODE 231

2. Applications 233

2.1. Application to singular Cauchy problems 234

2.2. Application to 2D polar coordinate 235

3. Typical examples 236

4. Proofs 238

4.1. Homogeneity of the coefficients of partial differential
operators

4.2. Proof of Theorem 1.1 and Theorem $1.2 \quad 239$

4.3. Proof of Proposition 1.1 242

4.4. Proof of Proposition 1.2 and Proposition $1.3 \quad 243$

4.5. Proof of Proposition $1.4 \quad 244$

4.6. Proof of Theorem $1.3 \quad 244$ 
5. Appendix

5.1. Weighted homogeneous function

5.2. Faà di Bruno's formula

\section{Introduction and results}

\subsection{Framework of the reduction from PDE to ODE}

The reductions of partial differential equations to ordinary differential ones by means of a homogeneity with respect to two variables are known as in Darboux's book [D].

First of all, as a typical example, let us consider a homogeneous partial differential equation:

$$
A_{n}[u(t, x)]=\left(\partial_{t}^{2}-t^{2 n} \partial_{x}^{2}-t^{n-1} \partial_{x}\right)[u(t, x)]=0
$$

for $n \geq 1$. We introduce the change of variables from $(t, x)$ to $(z, w)$ :

$$
\left\{\begin{array}{l}
z=z(t, x)=\frac{x}{t^{n+1}}, \\
w=w(t, x),
\end{array}\right.
$$

where $w(t, x)$ satisfies

$$
\left(t \partial_{t}+(n+1) x \partial_{x}\right) w(t, x) \neq 0
$$

This condition on $w(t, x)$ is imposed in order that Jacobian does not vanish. Then we have for a function $v(z)$ depending only on $z$

$$
A_{n}[v(z(t, x))]=t^{-2} \mathscr{A}_{n}[v(z)]
$$

where $\mathscr{A}_{n}$ is the ordinary differential operator with respect to $z$ expressed by

$$
\mathscr{A}_{n}=\left\{(n+1)^{2} z^{2}-1\right\} D_{z}^{2}+\{(n+1)(n+2) z-1\} D_{z}
$$

with a notation $D_{z}=d / d z$. The fact (1) produces sufficient benefit for us from the viewpoint of solving homogeneous partial differential equations. We can regard (1) as the reduction of the partial differential operator $A_{n}$ to the ordinary differential operator $\mathscr{A}_{n}$. Roughly speaking, the partial differential operator $A_{n}$ can be decomposed into the ordinary differential operator $\mathscr{A}_{n}$ with the multiplication operator $t^{-2}$ under $z=x / t^{n+1}$.

The purpose of this paper is the generalization of the reduction strategy of a partial differential operator to an ordinary differential operator in the sense of (1) and the characterization of the partial differential operator reducible to the ordinary differential operator under the reduction strategy. 


\subsubsection{Strategy of the reduction from PDE to ODE}

We treat a partial differential operators $P$ of order $m$ with holomorphic coefficients $b_{j, k}(t, x)$ which is written as

$$
\begin{aligned}
P\left(t, x, \partial_{t}, \partial_{x}\right) & =\sum_{j+k=0}^{m} b_{j, k}(t, x) \partial_{t}^{j} \partial_{x}^{k}, \\
b_{j, k}(t, x) & =\sum_{l, n \geq 0}\left(b_{j, k}^{l, n}\right) t^{l} x^{n} .
\end{aligned}
$$

For a complex constant $c$, we also consider a first order partial differential operator $Q_{c}$ :

$$
Q_{c}=t \partial_{t}+c x \partial_{x}
$$

Through our paper we adopt so-called Pochhammer symbol (rising factorial and falling factorial):

Definition 1.1. For $\alpha \in \boldsymbol{C}$, we define a rising factorial $(\alpha)^{k}$ and a falling factorial $(\alpha)_{k}$ with $k \in N \cup\{0\}$ as follows: In the case where $\alpha \neq 0$,

$$
(\alpha)^{k}=\frac{\Gamma(\alpha+k)}{\Gamma(\alpha)}, \quad(\alpha)_{k}=\frac{\Gamma(\alpha+1)}{\Gamma(\alpha-k+1)} .
$$

In the case where $\alpha=0$, we define $(0)^{0}=(0)_{0}=1(k=0)$ and $(0)^{k}=(0)_{k}=0$ $(k \in \boldsymbol{N})$.

The result which guarantees the generalization of the example in $\S 1.1$ is the following theorem:

Theorem 1.1. For some complex constant $c$, let us consider the change of variables $(z(t, x), w(t, x))=\left(x / t^{c}, w(t, x)\right)$ satisfying $Q_{c}[w(t, x)] \neq 0 . \quad$ In order that there exist a complex constants $d$ and an ordinary differential operator $\mathscr{P}$ for a partial differential operator $P$ such that

$$
P[v(z(t, x))]=t^{-d} \mathscr{P}[v(z)],
$$

the condition (CR) that

$$
\left[P, Q_{c}\right]=P Q_{c}-Q_{c} P=d P
$$

is the necessary and sufficient.

Corollary 1.1. The ordinary differential operator $\mathscr{P}$ in (5) is given explicitly by

$$
\mathscr{P}=\sum_{j+k=0}^{m}(-1)^{j} b_{j, k}(1, z) \sum_{p=0}^{j}(j)_{p}(c k)^{j-p} \sum_{\Lambda_{p}}\left[\prod_{q=1}^{p} \frac{1}{\kappa_{q} !}\left(\frac{(c)^{q}}{q !}\right)^{\kappa_{q}}\right] z^{\kappa} D_{z}^{k+\kappa},
$$


where the index set $\Lambda_{p}$ is the set of partition number of $p \in N$, that is

$$
\Lambda_{p}=\left\{\left(\kappa_{1}, \kappa_{2}, \ldots \kappa_{p}\right) ; \kappa_{j} \geq 0,1 \cdot \kappa_{1}+2 \cdot \kappa_{2}+\cdots+p \cdot \kappa_{p}=p\right\}
$$

and $\kappa$ is the total indices of $\left\{\kappa_{j}\right\}_{j=1}^{p}$, that is $\kappa=\sum_{j=1}^{p} \kappa_{j}$. In the case where $p=0$, that is $\Lambda_{0}=\varnothing$, the final sum is equivalent to

$$
\sum_{\Lambda_{p}}\left[\prod_{q=1}^{p} \frac{1}{\kappa_{q} !}\left(\frac{(c)^{q}}{q !}\right)^{\kappa_{q}}\right] z^{\kappa} D_{z}^{k+\kappa}=D_{z}^{k} .
$$

In particular, the homogeneous part of order $m$ of $\mathscr{P}$ is given explicitly by (8).

Remark 1.1. In the case $c=0$, the partial differential operator satisfying (CR) is of the form:

$$
P=t^{-d} \sum_{j+k=0}^{m} c_{j, k}(x) t^{j} \partial_{t}^{j} \partial_{x}^{k}
$$

where $c_{j, k}(x)$ is some function depending only on $x$. The ordinary differential operator guaranteed by Theorem 1.1 is expressed by

$$
\mathscr{P}=\sum_{k=0}^{m} c_{0, k}(z) D_{z}^{k} .
$$

From the analyticity of the coefficients $b_{j, k}(t, x)$, the commutation relation (CR) is equivalent to the condition that $b_{j, k}^{l, n}$ vanishes unless

$$
l+c n=j+c k-d
$$

holds. The identity (7) is coursed by the homogeneity of $b_{j, k}(t, x)$, that is

$$
Q_{c}\left[b_{j, k}(t, x)\right]=(j+c k-d) b_{j, k}(t, x) .
$$

Notice that $Q_{c}$ appears in Euler's homogeneous function theorem (See Appendix 5.1).

Corollary 1.2. Theorem 1.1 implies that we can express a solution $u(t, x)$ of the homogeneous partial differential equation $P[u]=0$ as $u(t, x)=v\left(x / t^{c}\right)$ with a solution of the homogeneous ordinary differential equation $\mathscr{P}[v]=0$.

Remark 1.2. The solution $u(t, x)$ of $P[u]=0$ in Corollary 1.2 is a homogeneous function of order 0 and weight $c$ with respect to the variable $t$, that is $u \in \operatorname{Ker} Q_{c}$. 


\subsubsection{Characterization of reducible partial differential operators}

In order to describe the strategy further, we denote by $L_{m}^{c, d}$ the class of the partial differential operators, which are determined by the commutation relation (CR), that is

$$
L_{m}^{c, d}=\left\{\sum_{j+k=0}^{m}\left(\sum_{l, n \geq 0}\left(b_{j, k}^{l, n}\right) t^{l} x^{n}\right) \partial_{t}^{j} \partial_{x}^{k} ; b_{j, k}^{l, n}=0 \text { unless } l+c n=j+c k-d\right\} .
$$

We list simple examples of $L_{m}^{c, d}$ in $\S 3$. Actually, when we assume that $c$ is positive integer and $d$ is non-negative integers, the coefficient $b_{j, k}(t, x)$ must be polynomial.

From (CR), one sees that the class $L_{m}^{c, d}$ is a vector space over $\boldsymbol{C}$. We can regard $L_{m}^{c, d}$ as a vector space generated by polynomials with complex coefficients with respect to four variables $t, x, \tau$ and $\xi$ in the symbol form.

Notice that the intersection of $L_{m}^{c, d}$ with respect to $c$ for a fixed $d$ is not empty generally. We denote the intersection class of $L_{m}^{c, d}$ with respect to $c$ by $L_{m}^{d}$, that is

$$
L_{m}^{d}=\bigcap_{c} L_{m}^{c, d}
$$

We call a partial differential operator belonging to $L_{m}^{d}$ a common operator. Unless a given partial differential operator $P$ does not belong to $L_{m}^{d}$, we can uniquely determine the constant $c$ and the class $L_{m}^{c, d}$ to which $P$ should belong.

We give the property of a common operator.

Proposition 1.1. For a common operator $P_{\text {com }} \in L_{m}^{d}$, we have

$$
\left[P_{\text {com }}, x \partial_{x}\right]=0, \quad\left[P_{\text {com }}, t \partial_{t}\right]=d P_{\text {com }} .
$$

Moreover $P_{\text {com }}$ can be expressed by the explicit form

$$
P_{\text {com }}=t^{-d} \sum_{\substack{0 \leq j+k \leq m \\ j-d \geq 0}}\left(b_{j, k}^{j-d, k}\right) t^{j} \partial_{t}^{j} x^{k} \partial_{x}^{k} .
$$

In particular, let $\mathscr{P}_{\text {com }}$ be the ordinary differential operator derived from $P_{\text {com }} \in L_{m}^{d}$ by Theorem 1.1. Then $\mathscr{P}_{\text {com }}$ is Euler type operator.

Similarly we denote the class of the ordinary differential operator $\mathscr{P}$ determined by Theorem 1.1 by $\mathscr{L}_{m}^{c, d}$ and we regard $\mathscr{L}_{m}^{c, d}$ as a vector space generated by polynomials with complex coefficients with respect to two variables $z$ and $\zeta$ in the symbol form. Theorem 1.1 defines the linear operator $T_{c}^{d}$ from 
$L_{m}^{c, d}$ to $\mathscr{L}_{m}^{c, d}$. Notice that the ordinary differential operator belonging to $\mathscr{L}_{m}^{c, d}$ is order at most $m$. In particular we see the following remark about $\operatorname{Ker} T_{c}^{d}$.

Remark 1.3. The kernel of $T_{c}^{d}$ is not empty. We can find the partial differential operator belonging to $\operatorname{Ker} T_{c}^{d}$. When $R$ is a partial differential operator satisfying (CR), one sees that the composition $R \circ Q_{c}$ belongs to $\operatorname{Ker} T_{c}^{d}$. For example, $Q_{c}$ itself, $\partial_{t} Q_{c}$ and $\partial_{x} Q_{c}$ belong to $\operatorname{Ker} T_{c}^{d}$.

The above considerations provide a whole strategy. If a partial differential operator of order $m$ is given, we can distinguish whether a given operator can be reduced to an ordinary differential operator or not. And moreover we investigate the class $L_{m}^{c, d}$, to which a given operator should belong. Namely we can determine the constants $c$ and $d$. In particular, the constants $c$ and $d$ can be done uniquely unless the given operator is a common operator. Consequently we can put $z=x / t^{c}$ and obtain the explicit form of the derived ordinary differential operator.

On the other hand, from an algebraic point of view, we obtain some algebras on commutation relations.

Proposition 1.2. Let $c, d_{1}$ and $d_{2}$ be complex constants. If partial differential operators $P_{1}$ and $P_{2}$ satisfy the commutation relations:

$$
\left[P_{1}, Q_{c}\right]=d_{1} P_{1}, \quad\left[P_{2}, Q_{c}\right]=d_{2} P_{2},
$$

then the compositions of $P_{1}$ and $P_{2}$ each other satisfy the following commutation relations

$$
\left\{\begin{array}{l}
{\left[\left(P_{1} \circ P_{2}\right), Q_{c}\right]=\left(d_{1}+d_{2}\right)\left(P_{1} \circ P_{2}\right),} \\
{\left[\left(P_{2} \circ P_{1}\right), Q_{c}\right]=\left(d_{1}+d_{2}\right)\left(P_{2} \circ P_{1}\right) .}
\end{array}\right.
$$

In the case where $P_{1}=P_{2}$ and $d_{1}=d_{2}$ hold, then

$$
\left[P_{1}^{k}, Q_{c}\right]=k d_{1} P_{1}^{k}
$$

is obtained recursively for $k \in N$.

Corollary 1.3. If reducible partial differential operators are given, we can generate reducible partial differential operators of higher order. Conversely, if a reducible partial differential operator is given, it may be decomposed into the product of two reducible operators of lower order. See such examples in $\S 4.4$.

When we denote the order of $P_{1}$ (resp. $P_{2}$ ) by $m_{1}$ (resp. $m_{2}$ ), Proposition 1.2 can be expressed formally by

$$
L_{m_{1}}^{c, d_{1}} \circ L_{m_{2}}^{c, d_{2}} \subset L_{m_{1}+m_{2}}^{c, d_{1}+d_{2}}
$$


in terms of the algebras on the classes $L_{m}^{c, d}$. In particular, the partial differential operator belonging to $L_{m}^{c, 0}$, that is commutative with respect to $Q_{c}$, generates partial differential operators of higher order preserving a constant $d$.

Proposition 1.3. If the commutation relations with the same constants $c$ and $d$ :

$$
\left[P_{1}, Q_{c}\right]=d P_{1} \quad\left[P_{2}, Q_{c}\right]=d P_{2}
$$

hold, then we get the following commutation relations:

$$
\left[\left(P_{1} \pm P_{2}\right), Q_{c}\right]=d\left(P_{1} \pm P_{2}\right) .
$$

Corollary 1.4. This proposition can be understood formally as

$$
L_{m_{1}}^{c, d} \cup L_{m_{2}}^{c, d} \subset L_{m_{1}}^{c, d} \quad\left(m_{1}>m_{2}\right)
$$

in terms of the algebras on the classes $L_{m}^{c, d}$. Namely, even if we add any partial differential operator belonging to $L_{m_{2}}^{c, d}$ to some partial differential one belonging to $L_{m_{1}}^{c, d}$, the partial differential operator remains belonging to the same class $L_{m_{1}}^{c, d}$.

\subsubsection{Succession of singularities from PDE to ODE}

We investigate the relation between the principal part of the derived ordinary differential operator $\mathscr{P}$ and that of the partial differential operator $P$. Let $\mathscr{P}_{m}$ be the principal part of $\mathscr{P}$ and $\operatorname{ch}(z)$ be its coefficient. Notice that, in Corollary 1.1, the set of the partition numbers which attain principal part is composed of $\left(\kappa_{1}, \kappa_{2}, \ldots, \kappa_{p}\right)=(p, 0, \ldots, 0)$ only. Therefore the principal part $\mathscr{P}_{m}$ can be expressed by

$$
\mathscr{P}_{m}=\left\{\sum_{j+k=m}(-c)^{j} b_{j, k}(1, z) z^{j}\right\} D_{z}^{m}=\operatorname{ch}(z) D_{z}^{m} .
$$

One sees that solutions of $\mathscr{P} v=0$ has singularities at the roots of the characteristic equation $\operatorname{ch}(z)=0$.

Remark 1.4. If every root of the characteristic equation is simple, then the ordinary differential operator $\mathscr{P}$ is Fuchsian type.

On the other hand, for a partial differential operator, we obtain the following proposition about the characteristic surfaces issuing from the origin.

Proposition 1.4. Put $\varphi(t, x)=x-\lambda t^{c}$ for $c>0$, where $\lambda$ is a root of the characteristic equation. Then the characteristic surface $\mathscr{S}$ of $P$ issuing from the origin is given by $\mathscr{S}=\{(t, x) ; \varphi(t, x)=0\}$. 
The constant $c$ means the tangential order between the characteristic surfaces. If $P$ is Fuchsian in the sense of [BG], the initial surface $\{(t, x) ; t=0\}$ is also such characteristic surface. Such a case can be treated in [WU1] and [WU2].

We can extend Theorem 1.1 to the case where a solution has a ramified singularity.

Theorem 1.2. For some complex constant $c$, let us consider the change of variables $(z(t, x), w(t, x))=\left(x / t^{c}, w(t, x)\right)$ satisfying $Q_{c}[w(t, x)] \neq 0 . \quad$ In order that there exist some complex constants $d, \alpha$ and an ordinary differential operator $\mathscr{P}_{\alpha}$ for a partial differential operator $P$ such that

$$
P\left[x^{\alpha} v(z(t, x ; \alpha))\right]=t^{-d} x^{\alpha} \mathscr{P}_{\alpha}[v(z ; \alpha)],
$$

the condition (CR) is the necessary and sufficient.

Moreover we obtain the explicit expression of $\mathscr{P}_{\alpha}$ as follows:

$$
\begin{aligned}
\mathscr{P}_{\alpha}= & \sum_{j+k=0}^{m}(-1)^{j} b_{j, k}(1, z) \sum_{\tilde{k}=0}^{k}\left(\begin{array}{l}
k \\
\tilde{k}
\end{array}\right)(\alpha)_{k-\tilde{k}^{z}} z^{-k+\tilde{k}} \\
& \times \sum_{p=0}^{j}(j)_{p}(c \tilde{k})^{j-p} \sum_{\Lambda_{p}}\left[\prod_{q=1}^{p} \frac{1}{\kappa_{q} !}\left(\frac{(c)^{q}}{q !}\right)^{\kappa_{q}}\right] z^{\kappa} D_{z}^{\tilde{k}+\kappa},
\end{aligned}
$$

where $\Lambda_{p}$ and $\kappa$ are the same notation as Theorem 1.1 .

Corollary 1.5. We can express a solution $u(t, x)$ of the homogeneous partial differential equation $P[u]=0$ as $u(t, x)=x^{\alpha} v\left(x / t^{c} ; \alpha\right)$ by means of one of the homogeneous ordinary differential equation $\mathscr{P}_{\alpha}[v(z ; \alpha)]=0$.

Remark 1.5. The solution $u(t, x)$ of $P[u(t, x)]=0$ in Corollary 1.5 is a homogeneous function of order $\alpha$ and weight $c$ with respect to the variable $t$, that is $u \notin \operatorname{Ker} Q_{c}$ for $\alpha \neq 0$.

It is important that Theorem 1.2 can be applied to the case where $P[u]=0$ has a solution with a ramified singularity on the characteristic surface $\mathscr{S}=$ $\left\{(t, x) ; x-\lambda t^{c}=0\right\}$ in Proposition 1.4.

$$
\begin{aligned}
u(t, x ; \alpha) & =\left(x-\lambda t^{c}\right)^{\alpha} v\left(x / t^{c} ; \alpha\right), \\
& =x^{\alpha}\left(\frac{z-\lambda}{z}\right) v(z ; \alpha)=x^{\alpha} \tilde{v}(z ; \alpha) .
\end{aligned}
$$

Notice that $u(t, x ; \alpha)$ is a homogeneous function of order $\alpha$ and weight $c$ with respect to the variable $t$. More generally $\varphi(t, x)^{\alpha}=\left(x-\lambda t^{c}\right)^{\alpha}$ is a homogeneous function of order $\alpha$ and weight $c$ with respect to the variable $t$ and the 
multiplication operator $\left(x-\lambda t^{c}\right)^{\alpha}$ satisfies (CR) with $d=-\alpha c$ :

$$
\left[\left(x-\lambda t^{c}\right)^{\alpha}, Q_{c}\right]=-\alpha c\left(x-\lambda t^{c}\right)^{\alpha} .
$$

This equality gives the following theorem:

Theorem 1.3. Let $P$ be a partial differential operator satisfying $(\mathbf{C R})$ and $P_{\alpha}$ satisfying

$$
\left(x-\lambda t^{c}\right)^{\beta} P_{\alpha}=P \circ\left(x-\lambda t^{c}\right)^{\alpha}
$$

for any $\lambda \neq 0$. Then $P_{\alpha}$ satisfies the following commutation relation:

$$
\left[P_{\alpha}, Q_{c}\right]=(d-(\alpha-\beta) c) P_{\alpha} .
$$

Remark 1.6. The homogeneous equations $P_{\alpha} u=0$ and $P u=0$ have the same characteristic surfaces. A solution of $P_{\alpha} u=0$, which is different from one of $P u=0$, is homogeneous function of order $\alpha$ and weight $c$ with respect to $t$.

Similarly we denote the principal part of the ordinary differential operator $\mathscr{P}_{\alpha}$ by $\mathscr{P}_{\alpha, m}$. We notice that, in Theorem 1.2, the set of the partition numbers which attain principal part is composed of $\left(\kappa_{1}, \kappa_{2}, \ldots, \kappa_{p}\right)=(p, 0, \ldots, 0)$ only and then we take $p=j$ and $\tilde{k}=k$. Therefore the principal part $\mathscr{P}_{\alpha, m}$ can be expressed by

$$
\mathscr{P}_{\alpha, m}=\left\{\sum_{j+k=m}(-c)^{j} b_{j, k}(1, z) z^{j}\right\} D_{z}^{m}=\operatorname{ch}(z) D_{z}^{m} .
$$

Consequently the principal part of $\mathscr{P}_{\alpha, m}$ has the same form as that of $\mathscr{P}_{m}$. Namely the principal part of the ordinary differential operator derived along our strategy is independent of the ramification index $\alpha$. Of course the characteristic surfaces determined by the principal part of the derived ordinary differential operator is also independent of $\alpha$.

\section{Applications}

We give some applications of the strategy of our reduction discussed in $\S 1$. The main results are independent of the type of the partial differential operator (elliptic, hyperbolic and parabolic). In particular, the application to the analysis of the propagation of the singularities of a singular Cauchy problem for hyperbolic differential equations is important.

We cite a singular Cauchy problem for a Fuchsian partial differential equation in $\S 2.1$ and refer a partial differential equation with an inhomogeneous term depending only on the angle under two dimensional polar coordinate in $\S 2.2$. 


\subsection{Application to singular Cauchy problems}

A singular Cauchy problem, how the singularity of the Cauchy data propagates along the characteristic surfaces, is one of the typical problems. In order to investigate the singularities of solutions concretely, it is known that the method of majorization is valid. This method consists of two parts. The first part is to expand solutions by means of the sequences of auxiliary functions which describe the singularities coming from the principal part of the partial differential equation. The second part is to show the convergence of the formal series of those sequences.

Generally the second part has a technical difficulty depending on the auxiliary functions associated with the equation. On the other hand, applying our reduction strategy to the first part, we can treat various type of partial differential equations as in $[\mathrm{F}],[\mathrm{U}]$. We explain such an application by taking up the singular Cauchy problem for so-called Euler-Poisson-Darboux equation:

$$
L[u(t, x)]=t\left(\partial_{t}^{2}-\partial_{x}^{2}-\frac{\gamma}{t} \partial_{t}-a(t, x) \partial_{t}-b(t, x) \partial_{x}-c(t, x)\right) u(t, x)=0
$$

with holomorphic coefficients $a, b, c$ and some complex constant $\gamma$ as in [WU1]. The operator $L$ is Fuchsian type in the sense of $[\mathrm{BG}]$. One sees that $L$ itself does not satisfy (CR). Therefore we consider the partial differential operator $P$ which is a derived higher order parts satisfying (CR) and treat the following singular Cauchy problem

$$
\left\{\begin{array}{l}
P[u]=\left(t \partial_{t}^{2}-t \partial_{x}^{2}-\gamma \partial_{t}\right)[u(t, x)]=0, \\
u(0, x)=\frac{x^{\alpha}}{\Gamma(\alpha+1)},
\end{array}\right.
$$

where $\alpha$ is some complex constant. The Cauchy data called wave form is ramified at the origin. The Cauchy problem (11) is a characteristic Cauchy problem.

One sees that the partial differential operator $P$ belongs to $L_{2}^{1,1}$ by comparing it with the examples in $\S 3$. Hence we introduce the change of variable $z(t, x)=x / t$. In order to deal with not only the ramified singularity of the Cauchy data but also its propagations along the characteristic surface, we apply Theorem 1.2 and its extension on the characteristic surface of $P$. We obtain $P\left[(t+x)^{\alpha} v(z(t, x) ; \alpha)\right]=t^{-1}(t+x)^{\alpha} \mathscr{P}_{\alpha}[v(z ; \alpha)]$, where

$$
\mathscr{P}_{\alpha}=(z-1)^{2} D_{z}^{2}+\{(2+\gamma) z-2 \alpha\} D_{z}-\frac{\alpha \gamma}{1+z}
$$


Notice that $t^{-1}(t+x)=1+z$. Hence the reduced homogeneous ordinary differential equation: $t^{-1}(t+x)^{\alpha} \mathscr{P}_{\alpha}[v(z ; \alpha)]=0$ is rewritten as

$$
(t+x)^{\alpha-1}\left((z-1)(z+1)^{2} D_{z}^{2}+\{(2+\gamma) z-2 \alpha\}(z+1) D_{z}-\alpha \gamma\right)[v(z)]=0 .
$$

Put $w=2 /(1+z)$, that is $(1+z) D_{z}=-w D_{w}$. Then we have

$$
2(t+x)^{\alpha-1}\left(w(1-w) D_{w}^{2}+\left\{-\gamma+\left(\alpha+\frac{\gamma}{2}-1\right) w\right\} D_{w}-\frac{\alpha \gamma}{2}\right)[v(z)]=0 .
$$

The change of variable $w=2 /(1+z)$ is Möbius transform, which maps three regular singular points $\pm 1, \infty$ to $0,1, \infty$ as the following table.

\begin{tabular}{|c||c|c|c|c|c|}
\hline$t$ & $-x$ & & $x$ & & 0 \\
\hline$z$ & -1 & & 1 & & $\infty$ \\
\hline$w$ & $\infty$ & & 1 & & 0 \\
\hline
\end{tabular}

As this Möbius transform implies, the homogeneous differential equation for the derived operator (14) is Gauss's hypergeometric differential equation, and its solution can be expressed as $F(-\alpha,-\gamma / 2,-\gamma ; z)$ under the usual notation of Gauss's hypergeometric function.

Notice that there are several ways to derive $P$ from $L$ and to apply Theorem 1.1 or Theorem 1.2 to $P$ even if $P$ belongs to the same class. On the former one, it is a delicate problem how we should derive a singular part (for example Fuchsian part) from the lower order terms except the highest order terms. In our previous work [WU2] one sees that the lower term which is not Fuchsian part effects the singularity. On the latter one, (11) can not be reduced to the hypergeometric differential equation by applying Theorem 1.1. The way to reduce to (14) is the best way in the viewpoint of studying the propagation of singularities of the solutions to (11), because the singularities of the hypergeometric function is analyzed explicitly by means of the characteristic indices.

The application of our strategy to a singular Cauchy problem implies that there exists some class of partial differential operators that local structures of the solutions to partial differential equations on the universal covering space can be described by global structures of those to ordinary differential equations.

\subsection{Application to $2 \mathrm{D}$ polar coordinate}

The change of variables to the polar coordinate is one of typical transformations based on a homogeneity. Recall that the polar coordinate of $\boldsymbol{R}^{2}$ :

$$
\boldsymbol{R}^{2} \ni(t, x) \mapsto(r, \theta)=\left(\sqrt{t^{2}+x^{2}}, \arctan (x / t)\right) \in \boldsymbol{R}_{>0} \times \boldsymbol{R} .
$$


Notice that the radius $r(t, x)$ is a homogeneous function of order 1 and the angle $\theta(t, x)$ is one of order 0 . One sees that Jacobian of the change of variables does not vanish except the origin $(t, x)=(0,0)$.

A partial differential equation with an inhomogeneous term depending only on the angle:

$$
P[u(t, x)]=g(\arctan (x / t))
$$

can be regarded as the special case where $\alpha=0, c=1$ and $d=0$. If $P$ satisfies (CR), we can get a radius-free solution by solving the ordinary differential equation:

$$
\mathscr{P}_{0}[v(z)]=g(\arctan z),
$$

where $z=x / t$.

This application implies that the strategy of our reduction in the special case $c=1$ gives the solvability of a partial differential equation along the angular direction.

\section{Typical examples}

In this section, we give typical examples of Theorem 1.1 under a simple assumption. Actually one sees that $j, k, l$ and $n$ are non-negative integers. In particular, we assume that $c$ is a positive integer and $d$ a non-negative integer. Then the forms of the partial differential operators guaranteed by Theorem 1.1 are restricted to those of the operators with polynomial coefficients. Namely, when the pair of $(j, k)$ is given, the pair of $(l, n)$ is determined by Diophantine equation $l+c n=j+c k-d$.

One of the typical classes of non-trivial operators of first order is $L_{1}^{c, 0}$ for $c \geq 2$. The partial differential operators belonging to $L_{1}^{c, 0}$ are of the form

$$
\left(b_{1,0}^{1,0}\right) t \partial_{t}+\left\{\left(b_{0,1}^{c, 0}\right) t^{c}+\left(b_{0,1}^{0,1}\right) x\right\} \partial_{x}+\left(b_{0,0}^{0,0}\right)
$$

and their derived ordinary differential operators belonging to $\mathscr{L}_{1}^{c, 0}$ are of the form:

$$
\left[\left\{-\left(b_{1,0}^{1,0}\right) c+\left(b_{0,1}^{0,1}\right)\right\} z+\left(b_{0,1}^{c, 0}\right)\right] D+\left(b_{0,0}^{0,0}\right)
$$

In a particular case where the coefficient $\left(b_{0,1}^{c, 0}\right)$ vanishes, one sees that the operator $\left(b_{1,0}^{1,0}\right) t \partial_{t}+\left(b_{0,1}^{0,1}\right) x \partial_{x}+\left(b_{0,0}^{0,0}\right)$ corresponds to a common operator. Remark that the coefficients of the common operator which is independent of the parameter $c$ are restricted to the form $\left(b_{j, k}^{j, k}\right)$.

Similarly we refer the case of partial differential operators of order two. The partial differential operators belonging to the class $L_{2}^{c, 0}$ for $c \geq 2$ are of the form: 


$$
\begin{aligned}
& \left(b_{2,0}^{2,0}\right) t^{2} \partial_{t}^{2}+\left\{\left(b_{1,1}^{1+c, 0}\right) t^{1+c}+\left(b_{1,1}^{1,1}\right) t x\right\} \partial_{t} \partial_{x}+\left\{\left(b_{0,2}^{2 c, 0}\right) t^{2 c}+\left(b_{0,2}^{c, 1}\right) t^{c} x+\left(b_{0,2}^{0,2}\right) x^{2}\right\} \partial_{x}^{2} \\
& +\left(b_{1,0}^{1,0}\right) t \partial_{t}+\left\{\left(b_{0,1}^{c, 0}\right) t^{c}+\left(b_{0,1}^{0,1}\right) x\right\} \partial_{x}+\left(b_{0,0}^{0,0}\right)
\end{aligned}
$$

and their derived ordinary differential operators belonging to $\mathscr{L}_{2}^{c, 0}$ are of the form:

$$
\begin{aligned}
& {\left[\left\{\left(b_{2,0}^{2,0}\right) c^{2}-\left(b_{1,1}^{1,1}\right) c+\left(b_{0,2}^{0,2}\right)\right\} z^{2}-\left\{\left(b_{1,1}^{1+c, 0}\right) c-\left(b_{0,2}^{c, 1}\right)\right\} z+\left(b_{0,2}^{2 c, 0}\right)\right] D^{2}} \\
& +\left[\left\{\left(b_{2,0}^{2,0}\right) c^{2}+\left(\left(b_{2,0}^{2,0}\right)-\left(b_{1,1}^{1,1}\right)-\left(b_{1,0}^{1,0}\right)\right) c+\left(b_{0,1}^{0,1}\right)\right\} z\right. \\
& \left.\quad-\left\{\left(b_{1,1}^{1+c, 0}\right) c-\left(b_{0,1}^{c, 0}\right)\right\}\right] D+\left(b_{0,0}^{0,0}\right) .
\end{aligned}
$$

On the other hand, when $d$ is not zero, the polynomial coefficients are more restricted. We give the examples in the case $d=1,2$. The partial differential operators belonging to the class $L_{2}^{c, 1}$ have the following form:

$$
\begin{aligned}
& t^{-1}\left[\left(b_{2,0}^{1,0}\right) t^{2} \partial_{t}^{2}+\left\{\left(b_{1,1}^{c, 0}\right) t^{c+1}+\left(b_{1,1}^{0,1}\right) t x\right\} \partial_{t} \partial_{x}\right. \\
& \left.\quad+\left\{\left(b_{0,2}^{2 c-1,0}\right) t^{2 c}+\left(b_{0,2}^{c-1,1}\right) t^{c} x\right\} \partial_{x}^{2}+\left(b_{1,0}^{0,0}\right) t \partial_{t}+\left(b_{0,1}^{c-1,0}\right) t^{c} \partial_{x}\right]
\end{aligned}
$$

and their derived ordinary differential operators belonging to $\mathscr{L}_{2}^{c, 1}$ take the next form:

$$
\begin{aligned}
t^{-1}( & {\left[\left\{\left(b_{2,0}^{1,0}\right) c^{2}-\left(b_{1,1}^{0,1}\right) c\right\} z^{2}-\left\{\left(b_{1,1}^{c, 0}\right) c-\left(b_{0,2}^{c-1,1}\right)\right\} z+\left(b_{0,2}^{2 c-1,0}\right)\right] D^{2} } \\
& \left.+\left[\left\{\left(b_{2,0}^{1,0}\right) c^{2}+\left(\left(b_{2,0}^{1,0}\right)-\left(b_{1,0}^{0,0}\right)\right) c-\left(b_{1,1}^{0,1}\right)\right\} z-\left\{\left(b_{1,1}^{c, 0}\right) c-\left(b_{0,1}^{c-1,0}\right)\right\}\right] D\right) .
\end{aligned}
$$

The forms of the partial differential operators, which belong to the class $L_{2}^{c, 2}$ for $c \geq 2$, are restricted to

$$
t^{-2}\left[\left(b_{2,0}^{0,0}\right) t^{2} \partial_{t}^{2}+\left(b_{1,1}^{c-1,0}\right) t^{c+1} \partial_{t} \partial_{x}+\left\{\left(b_{0,2}^{2 c-2,0}\right) t^{2 c}+\left(b_{0,2}^{c-2,1}\right) t^{c} x\right\} \partial_{x}^{2}+\left(b_{0,1}^{c-2,0}\right) t^{c} \partial_{x}\right]
$$

and those of their derived ordinary differential operators belonging to $\mathscr{L}_{2}^{c, 2}$ are

$$
\begin{gathered}
t^{-2}\left[\left(\left(b_{0,2}^{0,0}\right) c^{2} z^{2}+\left\{\left(b_{0,2}^{c-2,1}\right)-\left(b_{1,1}^{c-1,0}\right) c\right\} z+\left(b_{0,2}^{2 c-2,0}\right)\right) D^{2}\right. \\
\left.+\left(\left(b_{0,2}^{0,0}\right) c(1-c) z+\left\{\left(b_{0,1}^{c-2,0}\right)-\left(b_{1,1}^{c-1,0}\right) c\right\}\right) D\right] .
\end{gathered}
$$

For $c \geq d>2$, the partial differential operators belonging to $L_{2}^{c, d}$ and the ordinary ones belonging to $\mathscr{L}_{2}^{c, d}$ have the following forms:

$$
\begin{aligned}
& t^{-d}\left[\left(b_{1,1}^{1+c-d, 0}\right) t^{1+c, 0} \partial_{t} \partial_{x}+\left\{\left(b_{0,2}^{2 c-d, 0}\right) t^{2 c}+\left(b_{0,2}^{c-d, 1}\right) t^{c} x\right\} \partial_{x}^{2}+\left(b_{0,1}^{c-d, 0}\right) t^{c} \partial_{x}\right], \\
& t^{-d}\left[\left(\left\{\left(b_{0,2}^{c-d, 1}\right)-\left(b_{1,1}^{1+c-d, 0}\right) c\right\} z+\left(b_{0,2}^{2 c-d, 0}\right)\right) D^{2}+\left\{\left(b_{0,1}^{c-d, 0}\right)-\left(b_{1,1}^{1+c-d, 0}\right) c\right\} D\right] .
\end{aligned}
$$


From the above explicit representations of derived operators in the cases where $d$ is not zero, the coefficients of the higher order affect those of lower. Notice that the derived ordinary differential operator vanishes if these coefficients satisfy suitable relations. Hence we can confirm that $\operatorname{Ker} T_{c}^{d}$ is not empty.

\section{Proofs}

\subsection{Homogeneity of the coefficients of partial differential operators}

We first consider that what conditions are required in order that (CR) holds.

$$
\begin{gathered}
P \circ Q_{c}-Q_{c} \circ P=\sum_{j+k=0}^{m}\left[(j+c k) b_{j, k}(t, x)-Q_{c} b_{j, k}(t, x)\right] \partial_{t}^{j} \partial_{x}^{k}, \\
d P=\sum_{j+k=0}^{m} d b_{j, k}(t, x) \partial_{t}^{j} \partial_{x}^{k} .
\end{gathered}
$$

Therefore every variable coefficient $b_{j, k}(t, x)$ satisfies

$$
Q_{c} b_{j, k}(t, x)=(j+c k-d) b_{j, k}(t, x) .
$$

In the case $c \neq 0$, from Proposition 5.1, the equality (15) implies that each coefficient $b_{j, k}(t, x)$ is a homogeneous function of order $(j+c k-d) / c$ and weight $c$ with respect to the variable $t$. Hence Euler's homogeneous theorem for $b_{j, k}(t, x)$ holds, that is

$$
b_{j, k}\left(\lambda t, \lambda^{c} x\right)=\left(\lambda^{c}\right)^{\alpha} b_{j, k}(t, x)=\lambda^{j+c k-d} b_{j, k}(t, x) .
$$

Moreover, from the analyticity of $b_{j, k}(t, x)$, we obtain the coefficient $b_{j, k}^{l, n}$ vanishes unless the relation with respect to the power

$$
l+c n=j+c k-d
$$

holds. Actually, substituting $t^{-1}$ for $\lambda$ in the relation (16), we have

$$
b_{j, k}\left(1, \frac{x}{t^{c}}\right)=t^{-(j+c k-d)} b_{j, k}(t, x) .
$$

Put $z=x / t^{c}$, and then we obtain

$$
t^{d}\left(\frac{b_{j, k}(t, x)}{t^{j} x^{k}}\right)=z^{-k} b_{j, k}(1, z) .
$$

This equality plays an important role in the proof of main results. 
In the case $c=0,(15)$ is equivalent to $t \partial_{t} b_{j, k}(t, x)=(j-d) b_{j, k}(t, x)$. This differential equation can be solved as

$$
b_{j, k}(t, x)=c_{j, k}(x) t^{j-d}
$$

where $c_{j, k}(x)$ is some function depending only on $x$ and is the same as in Remark 1.1. Therefore the equality (18) is also valid in this case where $z=x / t^{0}$ as follows:

$$
t^{d}\left(\frac{b_{j, k}(t, x)}{t^{j} x^{k}}\right)=z^{-k} c_{j, k}(z)
$$

\subsection{Proof of Theorem 1.1 and Theorem 1.2}

First we assume the commutation relation (CR) and then we prove that there exist some complex constants $c \neq 0, d$ and an ordinary differential operator $\mathscr{P}$ for a partial differential operator $P$ such that

$$
P[v(z(t, x))]=t^{-d} \mathscr{P}[v(z)] .
$$

We notice that $z_{x}=t^{-c}$ is independent of the variable $x$. Hence one sees that $\partial_{x}^{k} v(z)=v^{(k)}(z) z_{x}^{k}$. Moreover we apply Libeniz formula to $\partial_{t}^{j}\left(v^{(k)}(z) z_{x}^{k}\right)$.

$$
\begin{aligned}
\partial_{t}^{j} \partial_{x}^{k} v(z) & =\sum_{p=0}^{j}\left(\begin{array}{c}
j \\
p
\end{array}\right)\left(\partial_{t}^{p} v^{(k)}(z)\right)\left(\partial_{t}^{(j-p)} t^{-c k}\right) \\
& =\sum_{p=0}^{j}(-1)^{j-p}\left(\begin{array}{l}
j \\
p
\end{array}\right)(c k)^{j-p} t^{-c k-j+p}\left(\partial_{t}^{p} v^{(k)}(z)\right) .
\end{aligned}
$$

We fix a variable $x$ and regard $\varphi(t)$ as $z(t)=x t^{-c}$ depending only on the variable $t$. Notice that $\partial_{t}^{j} z(t)=(-1)^{j}(c)_{j} x t^{-c-j}$. From Faà di Bruno's formula (See Appendix 5.2) we can calculate as

$$
\begin{aligned}
\partial_{t}^{p} v^{(k)}(z) & =\sum_{\Lambda_{p}} v^{(k+\kappa)}(z) \frac{(-1)^{p} p !}{\kappa_{1} ! \ldots \kappa_{p} !}\left[\left(\frac{\Gamma(c+1)}{\Gamma(c) \Gamma(2)}\right)^{\kappa_{1}} \cdots\left(\frac{\Gamma(c+p)}{\Gamma(c) \Gamma(p+1)}\right)^{\kappa_{p}}\right] x^{\kappa} t^{-c \kappa} t^{-p}, \\
& =(-1)^{p} p ! t^{-p} \sum_{\Lambda_{p}}\left[\prod_{q=1}^{p} \frac{1}{\kappa_{q} !}\left(\frac{(c)^{q}}{q !}\right)^{\kappa_{q}}\right] z^{\kappa} v^{(k+\kappa)}(z) .
\end{aligned}
$$

Hence we have

$$
\partial_{t}^{j} \partial_{x}^{k} v(z)=(-1)^{j} \sum_{p=0}^{j}(j)_{p}(c k)^{j-p} t^{-c k-j} \sum_{\Lambda_{p}}\left[\prod_{q=1}^{p} \frac{1}{\kappa_{q} !}\left(\frac{(c)^{q}}{q !}\right)^{\kappa_{q}}\right] z^{\kappa} v^{(k+\kappa)}(z)
$$


We shall study the partial differential operator $P$ by using (20).

$$
\begin{aligned}
P[v(z)] & =\sum_{j+k=0}^{m} b_{j, k}(t, x) \partial_{t}^{j} \partial_{x}^{k} v(z) \\
& =\sum_{j+k=0}^{m}(-1)^{j} b_{j, k}(t, x) \sum_{p=0}^{j}(j)_{p}(c k)^{j-p} t^{-c k-j} \sum_{\Lambda_{p}}\left[\prod_{q=1}^{p} \frac{1}{\kappa_{q} !}\left(\frac{(c)^{q}}{q !}\right)^{\kappa_{q}}\right] z^{\kappa} v^{(k+\kappa)}(z) .
\end{aligned}
$$

From (18), $P[v(z)]$ can be transformed as

$$
\begin{aligned}
P[v(z)]= & \sum_{j+k=0}^{m}(-1)^{j}\left(\frac{b_{j, k}(t, x)}{t^{j} x^{k}}\right) t^{-c k} x^{k} \\
& \times \sum_{p=0}^{j}(j)_{p}(c k)^{j-p} \sum_{\Lambda_{p}}\left[\prod_{q=1}^{p} \frac{1}{\kappa_{q} !}\left(\frac{(c)^{q}}{q !}\right)^{\kappa_{q}}\right] z^{\kappa} v^{(k+\kappa)}, \\
= & t^{-d}\left\{\sum_{j+k=0}^{m}(-1)^{j} b_{j, k}(1, z) \sum_{p=0}^{j}(j)_{p}(c k)^{j-p} \sum_{\Lambda_{p}}\left[\prod_{q=1}^{p} \frac{1}{\kappa_{q} !}\left(\frac{(c)^{q}}{q !}\right)^{\kappa_{q}}\right] z^{\kappa} v^{(k+\kappa)}\right\} .
\end{aligned}
$$

Therefore we obtain the ordinary differential operator $\mathscr{P}$ of order $m$ with respect to $z$, which is of the form:

$$
\mathscr{P}=\sum_{j+k=0}^{m}(-1)^{j} b_{j, k}(1, z) \sum_{p=0}^{j}(j)_{p}(c k)^{j-p} \sum_{\Lambda_{p}}\left[\prod_{q=1}^{p} \frac{1}{\kappa_{q} !}\left(\frac{(c)^{q}}{q !}\right)^{\kappa_{q}}\right] z^{\kappa} D_{z}^{k+\kappa}
$$

Similarly we can derive

$$
P\left[x^{\alpha} v(z(t, x ; \alpha))\right]=t^{-d} x^{\alpha} \mathscr{P}_{\alpha}[v(z ; \alpha)]
$$

from the commutation relation (CR) as follows:

$$
\begin{aligned}
P\left[x^{\alpha} f(z)\right] & =\sum_{j+k=0}^{m} b_{j, k}(t, x) \partial_{t}^{j} \partial_{x}^{k}\left(x^{\alpha} f(z)\right) \\
& =\sum_{j+k=0}^{m} b_{j, k}(t, x) \partial_{t}^{j}\left\{\sum_{\tilde{k}=0}^{k}\left(\begin{array}{l}
k \\
\tilde{k}
\end{array}\right)\left(\partial_{x}^{k-\tilde{k}} x^{\alpha}\right)\left(\partial_{x}^{\tilde{k}} f(z)\right)\right\} \\
& =\sum_{j+k=0}^{m} b_{j, k}(t, x) \sum_{\tilde{k}=0}^{k}\left(\begin{array}{l}
k \\
\tilde{k}
\end{array}\right)(\alpha)_{k-\tilde{k}} x^{\alpha-k+\tilde{k}} \partial_{t}^{j}\left(\partial_{x}^{\tilde{k}} f(z)\right)
\end{aligned}
$$


By applying the Faà di Bruno's formula (29) to $\partial_{t}^{j}\left(\partial_{x}^{\tilde{k}} f(z)\right)$, we have

$$
\begin{aligned}
P\left[x^{\alpha} f(z)\right]= & \sum_{j+k=0}^{m} b_{j, k}(t, x) \sum_{\tilde{k}=0}^{k}\left(\begin{array}{l}
k \\
\tilde{k}
\end{array}\right)(\alpha)_{k-\tilde{k}} x^{\alpha-k+\tilde{k}} \sum_{p=0}^{j}(-1)^{j-p}\left(\begin{array}{l}
j \\
p
\end{array}\right)(c \tilde{k})_{j-p} t^{-c \tilde{k}-j} \\
& \sum_{\Lambda_{p}} \frac{(-1)^{p} p !}{\kappa_{1} ! \kappa_{2} ! \ldots \kappa_{p} !}\left[\left(\frac{(c)_{1}}{1 !}\right)^{\kappa_{1}}\left(\frac{(c)_{2}}{2 !}\right)^{\kappa_{2}} \ldots\left(\frac{(c)_{p}}{l !}\right)^{\kappa_{p}}\right] z^{\kappa} f^{(\tilde{k}+\kappa)}(z), \\
= & x^{\alpha} \sum_{j+k=0}^{m}(-1)^{j}\left(\frac{b_{j, k}(t, x)}{t^{j} x^{k}}\right) \sum_{\tilde{k}=0}^{k}\left(\begin{array}{l}
k \\
\tilde{k}
\end{array}\right)(\alpha)_{k-\tilde{k}} z^{\tilde{k}} \\
& \sum_{p=0}^{j}(j)_{p}(c \tilde{k})_{j-p} \sum_{\Lambda_{l}}\left[\prod_{q=1}^{p} \frac{1}{\kappa_{q} !}\left(\frac{(c)^{q}}{q !}\right)^{\kappa_{q}}\right] z^{\kappa} f^{(\tilde{k}+k)}(z), \\
= & \frac{x^{\alpha}}{t^{d}}\left\{\sum_{j+k=0}^{m}(-1)^{j} b_{j, k}(1, z) \sum_{\tilde{k}=0}^{k}\left(\begin{array}{l}
k \\
k
\end{array}\right)(\alpha)_{k-\tilde{k}^{z}} z^{-k+\tilde{k}}\right. \\
& \left.\sum_{p=0}^{j}(j)_{p}(c \tilde{k})_{j-p} \sum_{\Lambda_{l}}\left[\prod_{q=1}^{p} \frac{1}{\kappa_{q} !}\left(\frac{(c)^{q}}{q !}\right)^{\kappa_{q}}\right] z^{\kappa} f^{(\tilde{k}+k)}(z)\right\} .
\end{aligned}
$$

Hence we have the explicit representation of the ordinary differential operator $\mathscr{P}_{\alpha}$ of order $m$ with respect to $z$, which is given by

$$
\begin{aligned}
\mathscr{P}_{\alpha}= & \sum_{j+k=0}^{m}(-1)^{j} b_{j, k}(1, z) \sum_{\tilde{k}=0}^{k}\left(\begin{array}{l}
k \\
\tilde{k}
\end{array}\right)(\alpha)_{k-\tilde{k}} z^{\tilde{k}-k} \\
& \sum_{p=0}^{j}(j)_{p}(c \tilde{k})_{j-p} \sum_{\Lambda_{l}}\left[\prod_{q=1}^{p} \frac{1}{\kappa_{q} !}\left(\frac{(c)^{q}}{q !}\right)^{\kappa_{q}}\right] z^{\kappa} D_{z}^{\tilde{k}+k} .
\end{aligned}
$$

Conversely, we suppose that there exist some complex constants $c \neq 0, d, \alpha$ and an ordinary differential operator $\mathscr{P}_{\alpha}$ for a partial differential operator $P$ such that

$$
P\left[x^{\alpha} v(z(t, x ; \alpha))\right]=t^{-d} x^{\alpha} \mathscr{P}_{\alpha}[v(z ; \alpha)] .
$$

Then let us show that the commutation relation (CR) holds. Remark that Theorem 1.2 under $\alpha=0$ is equivalent to Theorem 1.1. The following demonstration combines the proof of Theorem 1.1 with one of Theorem 1.2. Notice that the function $x^{\alpha} v\left(x / t^{c}\right)$ is a homogeneous function of order $\alpha$ and weight $c$ with respect to $t$, that is $Q_{c}\left[x^{\alpha} v\left(x / t^{c}\right)\right]=c \alpha\left(x^{\alpha} v\left(x / t^{c}\right)\right)$. Then we can calculate the commutator between $P$ and $Q_{c}$ as follows: 


$$
\begin{aligned}
{\left[P, Q_{c}\right]\left(x^{\alpha} v\left(\frac{x}{t^{c}}\right)\right) } & =P\left(c \alpha x^{\alpha} v\left(\frac{x}{t^{c}}\right)\right)-Q_{c}\left(x^{\alpha} t^{-d} \mathscr{P}_{\alpha}[v(z)]\right) \\
& =c \alpha P\left(x^{\alpha} v\left(\frac{x}{t^{c}}\right)\right)+d x^{\alpha} t^{-d} \mathscr{P}_{\alpha}[v(z)]-c \alpha x^{\alpha} t^{-d} \mathscr{P}_{\alpha}[v(z)] \\
& =d P\left(x^{\alpha} v\left(\frac{x}{t^{c}}\right)\right)
\end{aligned}
$$

Recall that the first example:

$$
A_{1}=\partial_{t}^{2}-t^{2} \partial_{x}^{2}-\partial_{x}
$$

belongs to a class $L_{2}^{2,2}$, because all coefficients of this operator satisfy the power relation $l+2 n=j+2 k-2$ and we check that $A_{1}$ is one of the next type partial differential operator

$$
\left(b_{2,0}^{0,0}\right) \partial_{t}^{2}+\left(b_{1,1}^{1,0}\right) t \partial_{t} \partial_{x}+\left\{\left(b_{0,2}^{2,0}\right) t^{2}+\left(b_{0,2}^{0,1}\right) x\right\} \partial_{x}^{2}+\left(b_{0,1}^{0,0}\right) \partial_{x} .
$$

Actually we can confirm the claim of Theorem 1.1.

$$
A_{1}^{1}[v(z(t, x))]=t^{-2} \mathscr{A}_{1}^{1}[v(z)]=t^{-2}\left[\left(4 z^{2}-1\right) D_{z}^{2}+(6 z-1) D_{z}\right] v(z) .
$$

\subsection{Proof of Proposition 1.1}

For $\tilde{c} \neq c$, a common operator $P_{\text {com }}$ satisfies (CR):

$$
P_{\text {com }} Q_{c}-Q_{c} P_{\text {com }}=d P_{\text {com }}, \quad P_{\text {com }} Q_{\tilde{c}}-Q_{\tilde{c}} P_{\text {com }}=d P_{\text {com }} .
$$

These commutation relations give us $(c-\tilde{c})\left[P_{c o m}, x \partial_{x}\right]=0$. Hence we have

$$
\left[P_{c o m}, x \partial_{x}\right]=0 \text {. }
$$

On the other hand, because $P_{c o m}$ satisfies (CR), we have

$$
\left[P_{c o m}, t \partial_{t}\right]+c\left[P_{c o m}, x \partial_{x}\right]=d P_{c o m} .
$$

Consequently we get

$$
\left[P_{c o m}, t \partial_{t}\right]=d P_{c o m}
$$

The commutation relation (21) is rewritten as

$$
\sum_{j+k=0}^{m}\left(k b_{j, k}-x\left(\partial_{x} b_{j, k}\right)\right) \partial_{t}^{j} \partial_{x}^{k}=0 .
$$


By the expression (3), the next equality holds.

$$
\sum_{l, n \geq 0}(k-n)\left(b_{j, k}^{l, n}\right) t^{l} x^{n}=0 .
$$

Hence the coefficients of a common operator $P_{\text {com }}$ satisfies $k=n$.

Similarly the commutation relation (22) is equivalent to

$$
\sum_{j=0}^{m} \sum_{k=0}^{m-j}\left(k b_{j, k}-x\left(\partial_{x} b_{j, k}\right)\right) \partial_{t}^{j} \partial_{x}^{k}=d P .
$$

From the expression (3), we have

$$
\sum_{l, n \geq 0}(j-l-d)\left(b_{j, k}^{l n}\right) t^{l} x^{n}=0 .
$$

The coefficients of a common operator $P_{\text {com }}$ also satisfies $l=j-d$. Hence we obtain

$$
P_{\text {com }}=t^{-d} \sum_{\substack{0 \leq j+k \leq m \\ j-d \geq 0}}\left(b_{j, k}^{j-d, k}\right) t^{j} \partial_{t}^{j} x^{k} \partial_{x}^{k} .
$$

The notation $\mathscr{D}_{z}$ stands for Euler operator $z D_{z}$. Then the following holds.

$$
\begin{aligned}
& t \partial_{t} v(z(t, x))=-c z D_{z} v(z)=-c \mathscr{D}_{z} v(z), \\
& x \partial_{x} v(z(t, x))=z D_{z} v(z)=\mathscr{D}_{z} v(z) .
\end{aligned}
$$

From the fact that $z^{k} D_{z}^{k}$ can be expressed by the linear combination of Euler operators $\left\{\mathscr{D}_{z}^{j}\right\}_{j=1}^{k}$, one sees that (23) can be reduced to Euler type operator.

\subsection{Proof of Proposition 1.2 and Proposition 1.3}

We show Proposition 1.2. From the assumptions, we can compute $\left[P_{1} P_{2}, Q_{c}\right]$ as follows:

$$
\begin{aligned}
P_{1} P_{2} Q_{c}-Q_{c} P_{1} P_{2} & =P_{1}\left(Q_{c} P_{2}+d_{2} P_{2}\right)-\left(P_{1} Q_{c}-d_{1} P_{1}\right) P_{2}, \\
& =\left(d_{1}+d_{2}\right) P_{1} P_{2} .
\end{aligned}
$$

Similarly we can prove the commutation relation for $P_{2} P_{1}$.

By means of the assumptions, we can show Proposition 1.3 directly

$$
\left(P_{1} \pm P_{2}\right) Q_{c}-Q_{c}\left(P_{1} \pm P_{2}\right)=d\left(P_{1} \pm P_{2}\right) \text {. }
$$


We check the claim of Proposition 1.2 and Proposition 1.3 by means of the first example $A_{1}$. Since the operator $A_{1} \in L_{2}^{2,2}$ satisfies the commutation relation

$$
\left[A_{2}^{1},\left(t \partial+2 x \partial_{x}\right)\right]=2 A_{2}^{1}
$$

its composition $\left(A_{1}\right)^{2}$ :

$$
\left(A_{1}\right)^{2}=\partial_{t}^{4}-2 t^{2} \partial_{t}^{2} \partial_{x}^{2}+t^{4} \partial_{x}^{4}-2 \partial_{t}^{2} \partial_{x}-8 t \partial_{t} \partial_{x}^{2}+2 t \partial_{x}^{3}-3 \partial_{x}^{2}
$$

satisfies the corresponding commutation relation:

$$
\left[\left(A_{1}\right)^{2},\left(t \partial+2 x \partial_{x}\right)\right]=4\left(A_{1}\right)^{2} .
$$

This commutation relation (24) means that $\left(A_{1}\right)^{2}$ belongs to $L_{4}^{2,4}$. On the other hand, $A_{1} \in L_{2}^{2,2}$ itself is generated by the composition of $P_{ \pm}=\partial_{t} \pm t \partial_{x} \in L_{1}^{2,1}$ as $A_{1}=P_{+} P_{-}$. However we remark that a similar operator $\tilde{A}_{1}$ dealt in [GS], [U]:

$$
\tilde{A}_{1}=\partial_{t}^{2}-\left(x+t^{2}\right) \partial_{x}^{2}-\partial_{x} \in L_{2}^{2,2}
$$

can not be generated by any composition of two partial differential operator of order one satisfying (CR).

\subsection{Proof of Proposition 1.4}

In order that the hypersurface given by $\varphi(t, x)=0$ is characteristic surface of $P$, the following should hold.

$$
\left.\sum_{j+k=m} b_{j, k}(t, x) \varphi_{t}^{j} \varphi_{x}^{k}\right|_{\mathscr{S}}=0 .
$$

We check (25) under $\varphi(t, x)=x-\lambda t^{c}$. Notice that $\varphi_{x}=1, \varphi_{t}=-c \lambda t^{c-1}$. We compute as follows:

$$
\begin{aligned}
\sum_{j+k=m} b_{j, k}(t, x) \varphi_{t}^{j} \varphi_{x}^{k} & =\sum_{j+k=m} b_{j, k}(t, x)(-c)^{j} \lambda^{j} t^{c j-j} \\
& =t^{-d} \sum_{j+k=m} b_{j, k}(1, z)(-c)^{j} \lambda^{j} t^{c j} x^{k} z^{-k} \\
& =t^{c m-d} \sum_{j+k=m} b_{j, k}(1, z)(-c)^{j} \lambda^{j}=t^{c m-d} \operatorname{ch}(\lambda) .
\end{aligned}
$$

Hence, if $\lambda$ is a root of the characteristic equation $\operatorname{ch}(z)=0$, (25) holds.

\subsection{Proof of Theorem 1.3}

Because $P$ satisfies (CR), we consider $\left[P, Q_{c}\right]\left(\varphi^{\alpha} u\right)$ :

$$
P Q_{c}\left(\varphi^{\alpha} u\right)-Q_{c} P\left(\varphi^{\alpha} u\right)=d P\left(\varphi^{\alpha} u\right) .
$$


From the commutation relation (10) and the assumption, we have

$$
\begin{aligned}
P\left\{\varphi^{\alpha}\left(Q_{c}+\alpha c\right) u\right\}-Q_{c}\left(\varphi^{\beta} P_{\alpha} u\right) & =d \varphi^{\beta} P_{\alpha} u, \\
\varphi^{\beta} P_{\alpha}\left(Q_{c}+\alpha c\right) u-\varphi^{\beta}\left(Q_{c}+\beta c\right) P_{\alpha} u & =d \varphi^{\beta} P_{\alpha} u, \\
P_{\alpha} Q_{c} u+\alpha c P_{\alpha} u-Q_{c} P_{\alpha} u-\beta c P_{\alpha} u & =d P_{\alpha} u, \\
P_{\alpha} Q_{c} u-Q_{c} P_{\alpha} u & =(d-(\alpha-\beta) c) P_{\alpha} u .
\end{aligned}
$$

Therefore we obtain $\left[P_{\alpha}, Q_{c}\right]=(d-(\alpha-\beta) c) P_{\alpha}$.

\section{Appendix}

\subsection{Weighted homogeneous function}

The necessary and sufficient condition (CR) given in Theorem 1.1 and Theorem 1.2 is based on a homogeneity. In this paper we recast so-called generalized homogeneous functions treated as in [HS] in line with our two variables case.

Let $c$ be a constant which expresses a weight between two variable and let $Q_{c}$ be a first order partial differential operator as

$$
Q_{c}=t \partial_{t}+c x \partial_{x}
$$

We shall introduce a weighted homogeneous function.

Definition 5.1. We say that $f(t, x)$ is a homogeneous function of order $\alpha$ and weight $c$ with respect to the variable $t$ if for any $\lambda$

$$
f\left(\lambda t, \lambda^{c} x\right)=\left(\lambda^{c}\right)^{\alpha} f(t, x)
$$

For the homogeneous function $f(t, x)$ defined above, the following proposition holds as well as Euler's homogeneous theorem.

Proposition 5.1. A function $f(t, x)$ is a homogeneous function of order $\alpha$ and weight $c$ with respect to the variable $t$ if and only if

$$
Q f(t, x)=(c \alpha) f(t, x) .
$$

Proof of Proposition 5.1. The proof of this proposition is the same as that of Euler's homogeneous function theorem. Differentiating (26) with respect to $\lambda$, we have

$$
t f_{t}\left(\lambda t, \lambda^{c} x\right)+c x \lambda^{c-1} f_{x}\left(\lambda t, \lambda^{c} x\right)=(c \alpha) \lambda^{(c \alpha-1)} f(t, x)
$$


The restriction of (28) to $\lambda=1$ coincides with (27). On the other hand, we show from (27) the three variables function $F(t, x, \lambda)=\lambda^{-c \alpha} f\left(\lambda t, \lambda^{c} x\right)$ is independent of $\lambda$. The fact gives the equality $F(t, x, \lambda)=F(t, x, 1)$ which is equivalent to (26). Actually one sees

$$
\partial_{\lambda} F(t, x, \lambda)=\lambda^{-c \alpha-1}\left(-c \alpha f\left(\lambda t, \lambda^{c} x\right)+\lambda t f_{t}\left(\lambda t, \lambda^{c} x\right)+c \lambda^{c} x f_{x}\left(\lambda t, \lambda^{c} x\right)\right)=0 .
$$

Remark that Proposition 5.1 can be extended naturally to the case where $f$ is a holomorphic function.

\subsection{Faà di Bruno's formula}

Lemma 5.1 (Faà di Bruno's formula $[\mathrm{R}]$ ). Let $f(z)$ and $\varphi(t)$ be holomorphic functions with $z=\varphi(t)$. For $F(t)=f(\varphi(t))$, the following holds

$$
F^{(p)}(t)=\sum_{\Lambda_{p}} f^{(\kappa)}(z) \frac{p !}{\kappa_{1} ! \kappa_{2} ! \ldots \kappa_{p} !}\left(\frac{\varphi^{(1)}(t)}{1 !}\right)^{\kappa_{1}}\left(\frac{\varphi^{(2)}(t)}{2 !}\right)^{\kappa_{2}} \ldots\left(\frac{\varphi^{(p)}(t)}{p !}\right)^{\kappa_{p}}
$$

where the index set $\Lambda_{p}$ is the set of partition numbers of $p \in \mathbf{N}$, that is

$$
\left\{\left(\kappa_{1}, \kappa_{2}, \ldots \kappa_{p}\right) \in \boldsymbol{Z}_{\geq 0}^{p} ; \kappa_{j} \geq 0, \sum_{j=1}^{p} j \kappa_{j}=p\right\}
$$

and $\kappa$ is the total indices $\left\{\kappa_{j}\right\}$, that is $\kappa=\sum_{j=1}^{p} \kappa_{j}$.

This formula is a generalized chain rule to higher derivatives. For example, we show the cases $p=1,2,3,4$ :

$$
\begin{aligned}
F^{(1)}(t)= & f^{(1)}(z) \varphi^{(1)}(t) \\
\Lambda_{1}= & \{(1)\} \\
F^{(2)}(t)= & f^{(2)}(z)\left(\varphi^{(1)}(t)\right)^{2}+f^{(1)}(z)\left(\varphi^{(2)}(t)\right)^{1} \\
\Lambda_{2}= & \{(2,0),(0,1)\} \\
F^{(3)}(t)= & f^{(3)}(z)\left(\varphi^{(1)}(t)\right)^{3}+3 f^{(2)}(z)\left(\varphi^{(1)}(t)\right)^{1}\left(\varphi^{(2)}(t)\right)^{1}+f^{(1)}(z)\left(\varphi^{(3)}(t)\right)^{1} \\
\Lambda_{3}= & \{(3,0,0),(1,1,0),(0,0,1)\} \\
F^{(4)}(t)= & f^{(4)}(z)\left(\varphi^{(1)}(t)\right)^{4}+6 f^{(3)}(z)\left(\varphi^{(1)}(t)\right)^{2}\left(\varphi^{(2)}(t)\right)^{1}+3 f^{(2)}(z)\left(\varphi^{(2)}(t)\right)^{2} \\
& +4 f^{(2)}(z)\left(\varphi^{(1)}(t)\right)^{1}\left(\varphi^{(3)}(t)\right)^{1}+f^{(1)}(z)\left(\varphi^{(4)}(t)\right)^{1} \\
\Lambda_{4}= & \{(4,0,0,0),(2,1,0,0),(1,0,1,0),(0,2,0,0),(0,0,0,1)\} .
\end{aligned}
$$




\section{References}

[BG] Baouendi, M. S. and Goulaouic, C., Cauchy problem with characteristic initial hypersurfaces, Comm. Pure Appl. Math., 26 (1973), 455-475.

[D] Darboux, G., Leçons sur la théorie générale des surfaces III, Les Grands Classiques Gauthier-Villars, Éditions Jacques Gabay, Sceaux, 1993.

[GS] Guillemin, V. and Schaeffer, D., On a certain class of Fuchsian partial differential equations, Duke Math. J., 44 (1977), 157-199.

[HS] Hankey, A. and Stanley, H. E., Systematic Application of Generalized Homogeneous Functions to Static Scaling, Dynamic Scaling, and Universality, Phys. Rev. B (3), 6 (1972), 3515-3542.

[R] Riordan, J., An introduction to combinatorial analysis, Dover Publications, Inc., Mineola, NY, 2002.

[U] Urabe, J., Singular Cauchy problem for a certain linear and 2nd order equation, J. Math. Kyoto Univ., 39 (1999), 1-24.

[F] Fujiié, S., Représentation hypergéométrique des singularités de la solutions du problème de Cauchy caractéristique à données holomorphes, Comm. Partial Differential Equations, 18 (1993), 1589-1629.

[WU1] Watanabe, T. and Urabe, J., A singular Cauchy problem for the Euler-Poisson-Darboux equation, J. Math. Pures Appl. (9), 93 (2010), 223-239.

[WU2] Watanabe, T. and Urabe, J., Singularities of solutions of the Fuchsian Cauchy problem with ramified data, preprint.

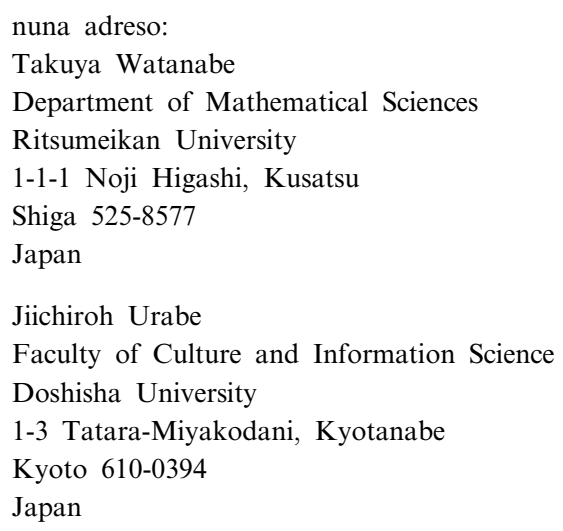

(Ricevita la 4-an de aprilo, 2012) 